\title{
Vacuum polarization in two-dimensional static spacetimes and dimensional reduction
}

\author{
Roberto Balbinot, Alessandro Fabbri.门 and Piero Nicolinif \\ Dipartimento di Fisica dell'Università di Bologna and INFN sezione di Bologna, \\ Via Irnerio 46, 40126 Bologna, Italy \\ Patrick J. Sutton \\ Center for Gravitational Wave Physics, \\ Center for Gravitational Physics and Geometry, \\ and Department of Physics, The Pennsylvania State University, \\ State College, PA, USA 16802-6300
}

\begin{abstract}
We obtain an analytic approximation for the effective action of a quantum scalar field in a general static two-dimensional spacetime. We apply this to the dilaton gravity model resulting from the spherical reduction of a massive, non-minimally coupled scalar field in the fourdimensional Schwarzschild geometry. Careful analysis near the event horizon shows the resulting two-dimensional system to be regular in the Hartle-Hawking state for general values of the field mass, coupling, and angular momentum, while at spatial infinity it reduces to a thermal gas at the black-hole temperature.
\end{abstract}

PACS numbers: 04.62.+v, 11.10.Gh, 11.10.Kk

*Electronic address: balbinot@bo.infn.it

${ }^{\dagger}$ Electronic address: fabbria@bo.infn.it

${ }^{\ddagger}$ Electronic address: nicolini@bo.infn.it

$\S$ Electronic address: psutton@gravity.phys.psu.edu 


\section{INTRODUCTION}

In a spacetime possessing a continuous symmetry one can decompose a quantum field into harmonics on the symmetrical subspace, effectively reducing the dimensionality of the system. For example, a quantum field in a four-dimensional spherically symmetric spacetime can be recast as a collection of two-dimensional fields labelled by their angular momentum. If one or a few of these modes make the dominant contribution to vacuum polarization in four dimensions, solving the reduced theory only for the modes of interest allows one to truncate "irrelevant" degrees of freedom.

This idea motivates much of the literature on two-dimensional black holes. By restricting attention to the spherically symmetric " $s$ mode" of a quantum field, which contributes the bulk of the Hawking radiation [1] emitted by Schwarzschild black holes, several authors [2, 3, 4, 5, 6] have proposed two-dimensional effective actions which could be used for the calculation of back-reaction effects and black-hole evaporation. These actions have difficulty, however, in reproducing the expected amount of Hawking radiation, in some cases predicting a negative flux (anti-evaporation) [7]. Before these actions can be trusted for back-reaction calculations, it is imperative to understand the behaviour of modes of fixed angular momentum in the Schwarzschild geometry.

A recent paper [8] partially addressed this question. Using point-splitting regularization and the well-known WKB approximation, the stress tensor due to the $s$ mode of a massless, minimally coupled quantum scalar field in the Schwarzschild geometry was found. A careful analysis demonstrated that the field is regular at the event horizon in the Hartle-Hawking state, and also exhibits the expected Hawking flux at infinity.

In this paper we extend the analysis of [8] to general two-dimensional static spacetimes and consider arbitrary harmonics of massive, nonminimally coupled scalar fields. We use the high-frequency approximation [10, 11, 12] to construct an effective action describing the quantum effects of a scalar field mode of fixed angular momentum at both zero and arbitrary finite temperature. This action is shown to yield a stress tensor which for the Schwarzschild spacetime coincides exactly with that obtained in canonical quantization through pointsplitting regularization using the WKB approximation for the modes. As before [8], cor-

rections to the WKB and high-frequency approximations are necessary to investigate the near-horizon behaviour. We show that the stress tensor is regular on the Schwarzschild 
horizon in the Hartle-Hawking state for arbitrary mass, coupling and angular momentum, and that it reduces to a thermal gas of massive particles at spatial infinity.

In Section II we review the quantum field-dilaton gravity model. Section [III briefly outlines the high-frequency approximation and applies it to our system, producing analytic expressions for the effective action and stress tensor throughout the spacetime. Special attention is devoted to the region near the horizon in Section $\mathbb{\Pi \nabla}$, where we demonstrate the regularity of the field in the Hartle-Hawking state. We conclude with some brief comments in section $\mathrm{V}$.

\section{QUANTUM FIELD COUPLED TO DILATON GRAVITY}

We wish to construct the two-dimensional theory corresponding to a mode of fixed angular momentum of a scalar field in a four-dimensional static [23] spherically symmetric geometry. The line element may be written as

$$
d s^{2}=h_{a b}\left(x^{c}\right) d x^{a} d x^{b}+\rho^{2} \mathrm{e}^{-2 \phi\left(x^{c}\right)} \Omega_{i j}\left(y^{k}\right) d y^{i} d y^{j},
$$

where $h_{a b}$ is a static two-dimensional Euclidean metric, $\Omega_{i j}$ is the metric of the unit twosphere, $\rho$ is a constant with dimensions of length, and $r \equiv \rho \mathrm{e}^{-\phi}$; the function $\phi$ is termed the dilaton. The four-dimensional scalar field $\widehat{\Phi}$ satisfies the equation

$$
\left(\square-m^{2}-\xi^{4} R\right) \widehat{\Phi}=0
$$

where $\square$ and ${ }^{4} R$ are the d'Alembertian and scalar curvature in four dimensions.

Our model is obtained by decomposing $\widehat{\Phi}$ in terms of spherical harmonics $Y_{l m}\left(y^{i}\right)$ on the two-sphere:

$$
\widehat{\Phi}\left(x^{c}, y^{k}\right)=\sum_{l=0}^{\infty} \sum_{m=-l}^{l} \widehat{\varphi}_{l}\left(x^{c}\right) \frac{Y_{l m}\left(y^{i}\right)}{r} .
$$

The two-dimensional field $\widehat{\varphi}_{l}$ satisfies

$$
\left(\Delta-m^{2}-V\right) \widehat{\varphi}_{l}=0
$$

where $\Delta$ is the d'Alembertian for the metric $h_{a b}$, the potential $V$ is

$$
V=\xi R+\frac{1}{r^{2}}[2 \xi+l(l+1)]+(4 \xi-1) \Delta \phi+(1-6 \xi)(\nabla \phi)^{2},
$$


and $R$ is the two-dimensional scalar curvature associated with $h_{a b}$. Equations (2.4)-(2.5) define the $2 \mathrm{D}$ quantum field theory which we will study. The special case $m=0, \xi=0$, $l=0$ in a Schwarzschild geometry was dealt with in [8] (see also [2, 3, 5, 6]).

In Section [II] we use the high-frequency approximation to calculate the one-loop effective action $W$ for (2.4)-(2.5). Given $W$, the expectation value of the stress tensor can be obtained as

$$
\left\langle T_{a b}\right\rangle=\frac{2}{\sqrt{h}} \frac{\delta W}{\delta h^{a b}} .
$$

This two-dimensional stress tensor is not conserved [5, 10]; rather, it satisfies

$$
\nabla_{a}\left\langle T^{a b}\right\rangle=-2 \nabla^{b} \phi P
$$

where the "effective pressure" $P$ corresponds to $\left\langle T_{\theta}{ }^{\theta}\right\rangle$ from four dimensions. This pressure is also easily calculated from the effective action:

$$
P=\frac{1}{2 \sqrt{h}} \frac{\delta W}{\delta \phi}
$$

Equations (2.7), (2.8) follow from the conservation of the full four-dimensional stress tensor. Using the relationship (2.3) between the four-dimensional field $\widehat{\Phi}$ and its two-dimensional counterpart $\widehat{\varphi}_{l}$, one can express the four-dimensional stress tensor in terms of the twodimensional one. Substitution into the four-dimensional conservation equation then yields (2.7). For details, see [5, 9, 10].

Local observables may also be calculated using the Euclidean Green function, which satisfies

$$
\left(\Delta-m^{2}-V\right) G(x, x)=-\frac{\delta\left(x-x^{\prime}\right)}{\sqrt{h}}
$$

and is related to the effective action by the formal expression

$$
W=-\frac{1}{2} \operatorname{Tr} \ln G
$$

The stress tensor and pressure can be obtained by applying certain differential operators to the Green function:

$$
\begin{gathered}
\left\langle T_{a b}\right\rangle=\lim _{x^{\prime} \rightarrow x} D_{a b} G\left(x, x^{\prime}\right), \\
P=\lim _{x^{\prime} \rightarrow x} D G\left(x, x^{\prime}\right),
\end{gathered}
$$


where [10]

$$
\begin{aligned}
D_{a b} \equiv & \left(\frac{1}{2}-\xi\right)\left(h_{a}{ }^{c^{\prime}} \nabla_{c^{\prime}} \nabla_{b}+h_{b}{ }^{c^{\prime}} \nabla_{a} \nabla_{c^{\prime}}\right)-\xi\left(\nabla_{a} \nabla_{b}+h_{a}{ }^{c^{\prime}} h_{b}{ }^{d^{\prime}} \nabla_{c^{\prime}} \nabla_{d^{\prime}}\right) \\
& +\left(2 \xi-\frac{1}{2}\right) h_{a b} h^{c d^{\prime}} \nabla_{c} \nabla_{d^{\prime}}+\xi R_{a b}+\left(2 \xi-\frac{1}{2}\right) h_{a b} \phi^{c}\left(\nabla_{c}+h_{c}^{d^{\prime}} \nabla_{d^{\prime}}\right) \\
& -\left(2 \xi-\frac{1}{2}\right)\left[\phi_{a}\left(\nabla_{b}+h_{b}^{d^{\prime}} \nabla_{d^{\prime}}\right)+\phi_{b}\left(\nabla_{a}+h_{a}^{d^{\prime}} \nabla_{d^{\prime}}\right)\right]+(1-6 \xi) \phi_{a} \phi_{b} \\
& +\left(2 \xi-\frac{1}{2}\right) h_{a b}\left[m^{2}+\xi R+4 \xi \Delta \phi+(1-6 \xi)(\nabla \phi)^{2}+\frac{1}{r^{2}}(2 \xi+l(l+1))\right] \\
D \equiv(2 \xi & \left.-\frac{1}{2}\right) h^{c d^{\prime}} \nabla_{c} \nabla_{d^{\prime}}-\frac{1}{2}(1-6 \xi)\left[\phi^{c}\left(\nabla_{c}+h_{c}^{d^{\prime}} \nabla_{d^{\prime}}\right)+\Delta \phi\right] \\
+ & \left(2 \xi-\frac{1}{2}\right)\left[m^{2}+\xi R+(4 \xi-1) \Delta \phi+(1-6 \xi)(\nabla \phi)^{2}\right]+\frac{2 \xi}{r^{2}}(2 \xi+l(l+1))
\end{aligned}
$$

Here $h_{a}{ }^{b^{\prime}}$ is the bivector of parallel transport, and $\phi_{a} \equiv \phi_{; a}$. This technique will be used to calculate corrections to the high-frequency approximation near the event horizon in Section IV.

\section{HIGH-FREQUENCY APPROXIMATION IN DILATON GRAVITY}

The high-frequency approximation [10, 11, 12] is a new generalized approximation scheme for quantum fields in static spacetimes. Here we apply this scheme to our dilaton gravity model (2.4)-(2.5). In Appendix $\mathrm{A}$ we show that for the $s$ mode $(l=0)$ in the reduced Schwarzschild spacetime our high-frequency approximation is equivalent to the WKB approximation; however, the high-frequency approximation is simpler and gives the effective action for general static spacetimes and field modes. Our analysis will be brief; for additional details see 110, 11, 12.

\section{A. High-Frequency Approximation in Two Dimensions}

The high-frequency approximation models the observable of interest, in this case the

effective action, using the ultraviolet divergences of the theory. Since high frequencies are equivalent to short distances, one is in essence assuming that the dominant features of the field (aside from any temperature dependence) can be approximated using its local behaviour.

The procedure is simple: 
1. Use as the starting point the Schwinger-DeWitt short-distance expansion [13, 14] for the observable of interest, which is a local expression containing all of the ultraviolet divergences of the theory. Since only this ultraviolet behaviour is of interest, discard any terms in the expansion which are finite in the coincidence limit.

2. Fourier transform over the time splitting, expand in a Laurent series in the inverse frequency $\omega^{-1}$ as $\omega \rightarrow \infty$, and retain only those terms which diverge when summed over $\omega$. (These are the only terms which are unambiguously specified by the ultraviolet divergences of the theory.) This defines the high-frequency approximation in the frequency domain.

3. Return to the time domain by summing over all frequencies (performing the inverse Fourier transform).

4. Renormalize in the usual way for point splitting, by subtracting the Schwinger-DeWitt expansion.

Let us apply this scheme to our dilaton-gravity model. We may write the line element of a two-dimensional static spacetime as

$$
d s^{2}=h_{a b} d x^{a} d x^{b}=\mathrm{e}^{-4 \eta(x)} d t^{2}+d x^{2},
$$

where $\eta$ is a function of the coordinate $x$ but not of $t$. Then for infinitesimally separated points, the Schwinger-DeWitt expansion [13, 14] for the Lagrangian of the effective action (2.10) is

$$
\begin{aligned}
L_{\mathrm{DS}}\left(t, x ; t^{\prime}, x^{\prime}\right)= & -\frac{1}{2 \pi\left(\tau^{2}+\epsilon^{2}\right)}+\frac{1}{8 \pi}\left(\frac{1}{6} R-V-m^{2}\right) \ln \frac{m^{2}\left(\tau^{2}+\epsilon^{2}\right)}{4 \mathrm{e}^{-2 \gamma}} \\
& +\frac{m^{2}}{8 \pi}-\frac{1}{12 \pi} \Delta \eta-\frac{1}{6 \pi}(\nabla \eta)^{2}+\mathcal{O}\left(\tau^{2}, \epsilon^{2}\right),
\end{aligned}
$$

where $\tau^{2}=\mathrm{e}^{-4 \eta}\left(t-t^{\prime}\right)^{2}, \epsilon=\left(x-x^{\prime}\right)^{2}$, and $\gamma$ is the Euler-Mascheroni constant.

To construct our approximation, we Fourier transform over the time splitting:

$$
L\left(x ; x^{\prime} \mid \omega\right)=\int_{-\infty}^{\infty} d t \mathrm{e}^{i \omega t} L\left(t, x ; 0, x^{\prime}\right) .
$$

Since we are only interested in the high-frequency limit of $L\left(x ; x^{\prime} \mid \omega\right)$, we need accurately model only the divergences of $L\left(t, x ; t^{\prime}, x^{\prime}\right)$. Casting off terms in (3.2) which are finite in the 
coincidence limit [24] and using equations (3.723.2) and (4.382.3) of [15], we arrive at [25]

$$
L_{\mathrm{HFA}}^{\mathrm{bare}}(x ; x \mid \omega) \equiv \frac{|\omega|}{2 \chi^{2}}-\frac{1}{4|\omega|}\left(\frac{1}{6} R-V-m^{2}\right),
$$

where $\chi^{2}=\mathrm{e}^{-4 \eta}$ is the norm of the Killing vector $\chi^{a}$ for the static space (3.1), and we have dropped all terms of $\mathcal{O}\left(\omega^{-3}\right)$ and higher.

We return to the time domain by summing (3.4) over all frequencies. For a scalar field at finite temperature $T=\beta^{-1}$ the allowed frequencies are discrete,

$$
\omega_{n}=n \frac{2 \pi}{\beta}
$$

where $n$ is an integer. (From (3.5), it is clear that the high-frequency approximation includes an assumption of high temperature.) The bare approximate effective Lagrangian in the time domain can then be evaluated using equation (E1) of [16], giving

$$
\begin{aligned}
L_{\mathrm{HFA}}^{\mathrm{bare}}\left(t, x ; t^{\prime}, x\right) & =\frac{2}{\beta} \sum_{n=1}^{\infty} \cos \left(\omega_{n}\left(t-t^{\prime}\right)\right) L_{\mathrm{HFA}}^{\mathrm{bare}}\left(x ; x \mid \omega_{n}\right) \\
& =-\frac{1}{2 \pi \tau^{2}}-\frac{\pi}{6 \chi^{2} \beta^{2}}+\frac{1}{8 \pi}\left(\frac{1}{6} R-V-m^{2}\right) \ln \frac{4 \pi^{2} \tau^{2}}{\chi^{2} \beta^{2}}
\end{aligned}
$$

Note that the zero-frequency mode makes no contribution in the high-frequency approximation; from (3.4)-(3.5), it consists of one term proportional to $n$, which vanishes, and one term proportional to $1 / n$, which must be dropped to regularize the infrared behaviour of the Lagrangian.

The ultraviolet divergences are renormalized in the usual way by subtracting the Schwinger-DeWitt expansion (3.2); integrating over the spacetime then gives the renormalized effective action

$$
W_{\mathrm{HFA}}^{\mathrm{ren}}=\int d^{2} x \sqrt{h}\left[-\frac{\pi}{6 \chi^{2} \beta^{2}}-\frac{m^{2}}{8 \pi}+\frac{(\nabla \eta)^{2}}{6 \pi}-\frac{1}{8 \pi} \ln \left(\frac{m^{2} \beta^{2} \chi^{2}}{16 \pi^{2} \mathrm{e}^{-2 \gamma}}\right)\left(\frac{1}{6} R-V-m^{2}\right)\right],
$$

where we have discarded a surface term. This action can be put into explicitly covariant form by the substitution

$$
\eta=-\frac{1}{4} \ln \chi^{2}
$$

All further expectation values will be calculated from $W_{\mathrm{HFA}}^{\mathrm{ren}}$ and hence will be renormalized already, so the superscript 'ren' will be dropped. 
To determine the stress tensor we must specify the potential $V$. Using (2.5), (2.6), and (2.8), we find

$$
\begin{aligned}
\left\langle T_{a b}\right\rangle_{\mathrm{HFA}}= & \frac{\pi}{6 \beta^{2} \chi^{2}}\left[h_{a b}-2 \frac{\chi_{a} \chi_{b}}{\chi^{2}}\right]+\frac{m^{2}}{8 \pi} h_{a b}-\frac{1}{6 \pi} h_{a b}(\nabla \eta)^{2}+\frac{1}{3 \pi} \nabla_{a} \eta \nabla_{b} \eta-\frac{1}{6 \pi} \frac{\chi_{a} \chi_{b}}{\chi^{2}} \Delta \eta \\
& +\frac{1}{4 \pi} \frac{\chi_{a} \chi_{b}}{\chi^{2}}\left[\left(\frac{1}{6}-\xi\right) R-m^{2}-\frac{1}{r^{2}}[2 \xi+l(l+1)]+(1-4 \xi) \Delta \phi+(6 \xi-1)(\nabla \phi)^{2}\right] \\
& -\frac{1}{2 \pi}\left[\left(\frac{1}{6}-\xi\right) 2\left[\nabla_{a} \nabla_{b} \eta-h_{a b} \Delta \eta\right]+(1-4 \xi)\left[\nabla_{a} \phi \nabla_{b} \eta+\nabla_{b} \phi \nabla_{a} \eta-h_{a b} \nabla \phi \cdot \nabla \eta\right]\right] \\
& +\frac{1}{8 \pi} \ln \left(\frac{m^{2} \beta^{2} \chi^{2}}{16 \pi^{2} \mathrm{e}^{-2 \gamma}}\right)\left[h_{a b}\left(-m^{2}-\frac{1}{r^{2}}[2 \xi+l(l+1)]+(6 \xi-1)(\nabla \phi)^{2}\right)\right. \\
& \left.+2(1-6 \xi) \nabla_{a} \phi \nabla_{b} \phi\right], \\
P_{\mathrm{HFA}}= & \frac{1}{8 \pi}[(1-4 \xi) 2 \Delta \eta+(1-6 \xi) 4 \nabla \phi \cdot \nabla \eta] \\
& +\frac{1}{8 \pi} \ln \left(\frac{m^{2} \beta^{2} \chi^{2}}{16 \pi^{2} \mathrm{e}^{-2 \gamma}}\right)\left[(6 \xi-1) \Delta \phi+\frac{1}{r^{2}}[2 \xi+l(l+1)]\right]
\end{aligned}
$$

where use of

$$
\frac{\delta \eta}{\delta h^{a b}}=\frac{\chi_{a} \chi_{b}}{4 \chi^{2}}
$$

has been made. One can verify that the stress tensor and pressure obey the "conservation" equation (2.7). For the conformally invariant theory $(m=0, \xi=0$, and $l=0)$, this stress tensor also has the correct anomalous trace [14] of

$$
\left\langle T_{\nu}{ }^{\nu}\right\rangle_{\mathrm{HFA}}=\frac{1}{4 \pi}\left(\frac{1}{6} R-V\right) .
$$

Equations (3.10)-(3.11) provide analytic expressions for expectation values for a wide class of quantum scalar fields in general static two-dimensional spacetimes. For example, for the Polyakov theory $(m=0, \xi=0, l=0, \phi=0)$ the effective action (3.8) reduces to the exact result for a static geometry [17]. Unfortunately, for more general systems these expectation values all contain $\ln \chi^{2}$, and thus typically will diverge at horizons, even in the Hartle-Hawking state. This appears to be a generic feature of local approximation schemes, occuring also in the WKB [16, 18], Page-Brown-Ottewill [19], and Killing [20] approximations in both two and four dimensions. In Section $\mathbb{\square}$ we shall see how the highfrequency approximation can be corrected near the horizon through more accurate modelling of the lowest-frequency field modes. 


\section{B. Spherically Reduced Schwarzschild Geometry}

For the special case of a field propagating in the Schwarzschild geometry,

$$
d s^{2}=f(r) d t^{2}+\frac{1}{f(r)} d r^{2}+r^{2}\left(d \theta^{2}+\sin ^{2} \theta d \phi^{2}\right), \quad f(r)=1-\frac{2 M}{r},
$$

we have

$$
\chi^{2}=f, \quad \eta=-\frac{1}{4} \ln f, \quad \phi=-\ln \frac{r}{\rho},
$$

where $\rho$ is some arbitrary length scale. The high-frequency approximation for the stress tensor and pressure for general $m, \xi$ and $l$ is

$$
\begin{aligned}
\left\langle T_{t}^{t}\right\rangle_{\mathrm{HFA}}= & -\frac{\pi}{6 \beta^{2} f}+\frac{1}{24 \pi r^{2} f}\left[-2 \frac{M}{r}+5 \frac{M^{2}}{r^{2}}+12 \xi\left(-4 \frac{M}{r}+7 \frac{M^{2}}{r^{2}}\right)\right]-\frac{m^{2}}{8 \pi}-\frac{l(l+1)}{4 \pi r^{2}} \\
& +\frac{1}{8 \pi r^{2}} \ln \left(\frac{m^{2} \beta^{2} f}{16 \pi^{2} \mathrm{e}^{-2 \gamma}}\right)\left[-\left(1-\frac{2 M}{r}\right)+4 \xi\left(1-\frac{3 M}{r}\right)-m^{2} r^{2}-l(l+1)\right], \\
\left\langle T_{r}{ }^{r}\right\rangle_{\mathrm{HFA}}= & \frac{\pi}{6 \beta^{2} f}+\frac{1}{24 \pi r^{2} f}\left[-6 \frac{M}{r}+11 \frac{M^{2}}{r^{2}}+12 \xi\left(2 \frac{M}{r}-3 \frac{M^{2}}{r^{2}}\right)\right]+\frac{m^{2}}{8 \pi} \\
& +\frac{1}{8 \pi r^{2}} \ln \left(\frac{m^{2} \beta^{2} f}{16 \pi^{2} \mathrm{e}^{-2 \gamma}}\right)\left[\left(1-\frac{2 M}{r}\right)+4 \xi\left(-2+\frac{3 M}{r}\right)-m^{2} r^{2}-l(l+1)\right], \\
P_{\mathrm{HFA}}= & \frac{1}{8 \pi r^{2}}\left[\frac{4 M}{r}-\xi^{20 M} \frac{r}{r}\right] \\
& +\frac{1}{8 \pi r^{2}} \ln \left(\frac{m^{2} \beta^{2} f}{16 \pi^{2} \mathrm{e}^{-2 \gamma}}\right)\left[-1+\frac{4 M}{r}+8 \xi\left(1-3 \frac{M}{r}\right)+l(l+1)\right] .
\end{aligned}
$$

For $l=0$ and general $m$ and $\xi$, these expectation values coincide with the WKB approximation, as shown in Appendix A. For a zero-temperature conformally invariant field $(\beta \rightarrow \infty$, $m=0, \xi=l=0$, with the $m$ in the logarithm terms replaced by an arbitrary cutoff param-

eter), (3.16) also coincides with the stress tensor and pressure obtained from integrating the two-dimensional conformal anomaly alone [5].

At spatial infinity, the stress tensor (3.16a), (3.16b) reduces precisely to that of a thermal gas of massive particles at high temperature in flat space, except that $\left\langle T_{r}^{r}\right\rangle$ is missing a term proportional to $m / \beta$. This term is lost because the high-frequency approximation does not include the contribution of the zero-frequency mode, which must be added by hand. Using the WKB approximation presented in Appendix A, one finds that at spatial infinity the only nonvanishing contribution of the $n=0$ mode is

$$
\left\langle T_{r}^{r}\right\rangle_{n=0 \mid W K B}=-\frac{m}{2 \beta \sqrt{f}} .
$$


Adding this term to (3.16b) reproduces exactly the stress tensor of a thermal gas of massive particles at high temperature in flat space. This stress tensor obeys the conservation equation (2.7), with the right-hand side of (2.7) vanishing at spatial infinity.

The situation at the horizon is more troublesome. It was seen in 8 that for the conformally invariant field in the Hartle-Hawking state $(\beta=8 \pi M)$ the stress tensor and pressure (3.16) diverge logarithmically at the event horizon in a freely falling frame [26] due to the logarithm terms. For nonconformal fields (any of $m, \xi$, or $l$ nonzero), the additional terms in (3.16) produce yet stronger divergences on the past and future horizons. Since the HartleHawking state should be regular on the horizon, this implies that the high-frequency and WKB approximations break down there. Some indication of why comes from inspection of (3.2), (3.7): the logarithm term in the bare Lagrangian (3.7) does not contain the norm of the Killing vector [note that $\tau^{2} / \chi^{2}=\left(t-t^{\prime}\right)^{2}$; the $\ln \chi^{2}$ terms arise purely from the renormalization counterterms (3.2). This implies that the bare high-frequency approximation misses contributions which go as $\ln \chi^{2}$ near the horizon. In the following section we demonstrate that this omission occurs because the high-frequency approximation fails to accurately model the lowest-frequency modes $(n \leq 2)$ near the horizon. Correcting for these modes shows that the stress tensor and pressure are indeed regular on the horizon in the Hartle-Hawking state.

\section{REGULARITY OF THE HARTLE-HAWKING STATE}

In this section we demonstrate the regularity of the stress tensor and pressure at the event horizon of the Schwarzschild geometry in the Hartle-Hawking state for general $m, \xi$, and l. We do this by solving the Green function equation (2.9) near the horizon for individual modes $\omega_{n}=2 \pi n / \beta$ of small $n$, and using these more accurate expansions to correct the high-frequency approximation. This analysis closely follows that of [8].

\section{A. Mode-by-Mode Predictions of the High-Frequency Approximation}

We begin by listing the contributions made by each frequency $n$ to expectation values in

the high-frequency approximation. This will be necessary later for determining the amount by which the approximation must be corrected for each mode. 
Using (3.4)-(3.6) one finds that for each $n>0$ the bare contributions to the stress tensor and pressure for $\beta=8 \pi M$ are

$$
\begin{aligned}
\left\langle T_{a}^{b}\right\rangle_{n \mid \mathrm{HFA}}= & \frac{n}{32 \pi M^{2} f}\left[2 \delta_{a}^{0} \delta_{0}^{b}-\delta_{a}^{b}\right] \\
& +\frac{1}{4 \pi n}\left[-\delta_{a}^{b}\left(m^{2}+\frac{\ell(\ell+1)}{r^{2}}+\frac{2 \xi}{r^{2}}\right)+(1-6 \xi) \frac{f}{r^{2}}\left(2 \delta_{a}^{r} \delta_{r}^{b}-\delta_{a}^{b}\right)\right], \\
P_{n \mid \mathrm{HFA}}= & \frac{1}{4 \pi r^{2} n}\left[(6 \xi-1)\left(1-\frac{4 M}{r}\right)+2 \xi+\ell(\ell+1)\right] .
\end{aligned}
$$

Note the absence of any logarithmic terms. Also, recall that the high-frequency approximation includes no contributions from the $n=0$ mode.

\section{B. Corrected Green Function near the Horizon}

We now proceed to calculate the exact contribution to the Green function and local observables made by each mode $n$ in the vicinity of the event horizon of the Schwarzschild geometry. We do this by solving for the Green function as a power series in the physical distance to the event horizon. We assume the field to be in the Hartle-Hawking state $(\beta=8 \pi M)$.

In the $(t, r)$ sector of the Schwarzschild spacetime (3.14) we decompose the Green function (2.9) into Fourier modes as

$$
G\left(t, r ; t^{\prime}, r^{\prime}\right)=\frac{1}{\beta\left[f(r) f\left(r^{\prime}\right)\right]^{\frac{1}{4}}}\left[G_{0}\left(r, r^{\prime}\right)+2 \sum_{n=1}^{\infty} \cos \left(\omega_{n}\left(t-t^{\prime}\right)\right) G_{n}\left(r, r^{\prime}\right)\right] .
$$

Substituting (4.2) and a similar decomposition for the delta function into the differential equation (2.9) for the two-dimensional Green function, and expanding in terms of the physical distance $L$ to the event horizon, where

$$
d L=\frac{d r}{f^{\frac{1}{2}}}
$$

the differential equation with $r \neq r^{\prime}$ becomes

$$
\partial_{L}^{2} G_{n}-\left(\frac{4 n^{2}-1}{4 L^{2}}+\frac{\alpha_{n}^{2}}{M^{2}}-\frac{A_{n}^{2}}{16 M^{4}} L^{2}+O\left(L^{4}\right)\right) G_{n}=0
$$

where we define the constants

$$
\alpha_{n}^{2} \equiv(m M)^{2}+\frac{l(l+1)}{4}+\frac{1}{6}+\frac{n^{2}}{12},
$$




$$
A_{n}^{2} \equiv \frac{n^{2}+56+60 l(l+1)}{120} .
$$

Neglecting the $O\left(L^{2}\right)$ term, (4.4) becomes a differential equation for the Bessel functions of imaginary argument. The solution for $G_{n}$ is easily shown to be

$$
G_{n}\left(r, r^{\prime}\right)=\left(L L^{\prime}\right)^{\frac{1}{2}} I_{n}\left(\frac{\alpha_{n} L_{<}}{M}\right) K_{n}\left(\frac{\alpha_{n} L_{>}}{M}\right),
$$

where $L_{>}\left(L_{<}\right)$is the physical distance to the greater (lesser) of $r, r^{\prime}$. This solution obeys the derivative condition resulting from integrating across the delta-function singularity at $r=r^{\prime}$.

The approximate solution (4.7) will yield the pressure accurately to $O(1+\ln L)=O(1+$ $\ln f$ ) inclusive, which contains all of the potentially divergent terms. To prove the finiteness of the stress tensor, however, it is also necessary to calculate the $O\left(L^{\frac{1}{2}} \ln L\right)=O(f \ln f)$ terms; a careful examination reveals that this requires taking the $O\left(L^{2}\right)=O(f)$ term in (4.4) into account. The simplest way to do this is to begin with the approximate solution (4.7), expand it for small $L$ and $L^{\prime}$, and modify the higher-order terms so that the resulting Green function satisfies (4.4) up to and including the $O\left(L^{2}\right)$ term. The resulting Green functions $G_{n}$ for $n=0,1,2,3$ are listed in Appendix B.

\section{Corrections to Local Observables}

We now calculate the precise contributions to the stress tensor and pressure for modes of small $n$, and use this information to correct the high-frequency approximation.

Using (2.11), for a contribution to the two-dimensional Green function of the form

$$
\cos \left(\omega_{n}\left(t-t^{\prime}\right)\right) F_{n}\left(r, r^{\prime}\right)
$$


the corresponding contributions to the stress tensor and pressure in $t, r$ coordinates are

$$
\begin{aligned}
\left\langle T_{t}^{t}\right\rangle_{n}=\lim _{r^{\prime} \rightarrow r}[ & \left(2 \xi+\frac{1}{2}\right) \frac{\omega_{n}^{2}}{f}+\xi \frac{2 M}{r^{3}}+\left(2 \xi-\frac{1}{2}\right)\left[m^{2}+\frac{f+l(l+1)}{r^{2}}\right] \\
& \left.+\left[\frac{1}{2} f+\xi\left(-2+\frac{3 M}{r}\right)\right] \frac{1}{r}\left(\partial_{r}+\partial_{r^{\prime}}\right)+\left(2 \xi-\frac{1}{2}\right) f \partial_{r} \partial_{r^{\prime}}\right] F_{n}\left(r, r^{\prime}\right), \\
\left\langle T_{r}^{r}\right\rangle_{n}=\lim _{r^{\prime} \rightarrow r}\left[\left(2 \xi-\frac{1}{2}\right) \frac{\omega_{n}^{2}}{f}+\xi \frac{2 M}{r^{3}}+\left(2 \xi-\frac{1}{2}\right)\left[m^{2}+\frac{f+l(l+1)}{r^{2}}\right]+(1-6 \xi) \frac{f}{r^{2}}\right. & \\
& \left.+\left[-\frac{1}{2} f+\xi\left(2-\frac{5 M}{r}\right)\right] \frac{1}{r}\left(\partial_{r}+\partial_{r^{\prime}}\right)+\frac{1}{2} f \partial_{r} \partial_{r^{\prime}}-\xi f\left(\partial_{r}^{2}+\partial_{r^{\prime}}^{2}\right)\right] F_{n}\left(r, r^{\prime}\right), \\
P_{n}=\lim _{r^{\prime} \rightarrow r}\left[\left(2 \xi-\frac{1}{2}\right) \frac{\omega_{n}^{2}}{f}+2 \xi \frac{f}{r^{2}}+\frac{l(l+1)}{2 r^{2}}+\left(2 \xi-\frac{1}{2}\right)\left[m^{2}+\frac{f+l(l+1)}{r^{2}}\right]\right. & \\
& \left.+(1-6 \xi) \frac{f}{2 r}\left(\partial_{r}+\partial_{r^{\prime}}\right)+\left(2 \xi-\frac{1}{2}\right) f \partial_{r} \partial_{r^{\prime}}\right] F_{n}\left(r, r^{\prime}\right) .
\end{aligned}
$$

Using the $G_{n}$ in Appendix B one can calculate the contribution to the stress tensor from each mode near the horizon. Subtracting the corresponding high-frequency approximation result (4.1) yields the corrections that should be added to the stress tensor and pressure for each $n$. Doing so, one finds that divergent corrections come only from the $n=0,1,2$ modes; higher modes give only corrections which are regular at the horizon. The total divergent corrections to be added to the high-frequency approximation are

$$
\begin{aligned}
\delta\left\langle T_{a}^{b}\right\rangle_{\text {div corr }}= & \frac{1}{96 \pi M^{2}}\left[\frac{3 \xi}{f}+\left(12 \mu^{2}+6 \xi+3 l(l+1)\right)+(3-18 \xi) f \ln f\right]\left(\begin{array}{rr}
1 & 0 \\
0 & -1
\end{array}\right) \\
& +\frac{1}{96 \pi M^{2}}\left(12 \mu^{2}+6 \xi+3 l(l+1)\right) \ln f\left(\begin{array}{ll}
1 & 0 \\
0 & 1
\end{array}\right) \\
\delta P_{\text {div corr }}= & \frac{1}{96 \pi M^{2}}(-3+12 \xi-3 l(l+1)) \ln f .
\end{aligned}
$$

For the conformal field these reproduce the divergent terms found in [8] (note that in [8] $P$ was defined with an additional factor of $\left.\left(4 \pi r^{2}\right)^{-1}\right)$. By adding these corrections to the high-frequency approximation (3.16) one finds that the resulting stress tensor and pressure are indeed regular on the event horizon for general $m, \xi$, and $l$.

The corrected stress tensor and pressure near the horizon, including all finite corrections 
for the $n=0,1,2$ modes, are

$$
\begin{aligned}
\left(1920 \pi M^{2}\right)\left\langle T_{t}^{t}\right\rangle= & -40-20 \xi-60 l(l+1)+80 \xi \mu^{2}+20 \xi l(l+1)+f\left(170+160 \mu^{2}\right. \\
& +220 l(l+1)-480 \mu^{4}-1334 \xi-1840 \xi \mu^{2}-720 \xi \mu^{4}-30 l^{2}(l+1)^{2} \\
& \left.-360 \xi \mu^{2} l(l+1)-1690 \xi l(l+1)-240 \mu^{2} l(l+1)-45 \xi l^{2}(l+1)^{2}\right) \\
& +\ln \left(1+\frac{3 l(l+1)+2}{12 \mu^{2}}\right)\left[240 \mu^{2}+60 l(l+1)-480 \xi \mu^{2}-120 \xi l(l+1)\right. \\
& +f\left(480 \mu^{2}+2880 \mu^{4}-1920 \xi \mu^{2}-8640 \xi \mu^{4}+180 l^{2}(l+1)^{2}\right. \\
& \left.\left.+1440 \mu^{2} l(l+1)-120 \xi l(l+1)-540 \xi l^{2}(l+1)^{2}-4320 \xi \mu^{2} l(l+1)\right)\right] \\
+ & \ln \left(1+\frac{l(l+1)+1}{4 \mu^{2}}\right)\left[120 \xi+480 \xi \mu^{2}+120 \xi l(l+1)+f\left(60-480 \mu^{2}\right.\right. \\
& -120 l(l+1)-2880 \mu^{4}+480 \xi+4800 \xi \mu^{2}+11520 \xi \mu^{4}+1200 \xi l(l+1) \\
+ & \left.\left.5760 \xi \mu^{2} l(l+1)+720 \xi l^{2}(l+1)^{2}-1440 \mu^{2} l(l+1)-180 l^{2}(l+1)^{2}\right)\right] \\
+ & \ln \left(1+\frac{l(l+1)+2}{4 \mu^{2}}\right)\left[f \left(-1080 \xi-2880 \xi \mu^{2}-2880 \xi \mu^{4}\right.\right. \\
& \left.\left.-180 \xi l^{2}(l+1)^{2}-1080 \xi l(l+1)-1440 \xi \mu^{2} l(l+1)\right)\right]+O\left(f^{2}\right), \quad(4.11 \mathrm{a})
\end{aligned}
$$

$$
\begin{aligned}
\left(1920 \pi M^{2}\right)\left\langle T_{r}^{r}\right\rangle= & -40-20 \xi-60 l(l+1)+80 \xi \mu^{2}+20 \xi l(l+1)+f(70+60 l(l+1) \\
& -160 \mu^{4}-498 \xi-400 \xi \mu^{2}-240 \xi \mu^{4}-120 \xi \mu^{2} l(l+1)-80 \mu^{2} l(l+1) \\
& \left.-10 l^{2}(l+1)^{2}-510 \xi l(l+1)-15 \xi l^{2}(l+1)^{2}\right) \\
& +\ln \left(1+\frac{3 l(l+1)+2}{12 \mu^{2}}\right)\left[240 \mu^{2}+60 l(l+1)-480 \xi \mu^{2}-120 \xi l(l+1)\right. \\
& +f\left(480 \mu^{2}+960 \mu^{4}-1920 \xi \mu^{2}-2880 \xi \mu^{4}-360 \xi l(l+1)\right. \\
& \left.\left.-180 \xi l^{2}(l+1)^{2}+480 \mu^{2} l(l+1)-1440 \xi \mu^{2} l(l+1)+60 l^{2}(l+1)^{2}\right)\right] \\
& +\ln \left(1+\frac{l(l+1)+1}{4 \mu^{2}}\right)\left[120 \xi+480 \xi \mu^{2}+120 \xi l(l+1)+f\left(-60-480 \mu^{2}\right.\right. \\
& -120 l(l+1)-960 \mu^{4}+480 \xi+2880 \xi \mu^{2}+3840 \xi \mu^{4}+720 \xi l(l+1) \\
& \left.\left.-60 l^{2}(l+1)^{2}+240 \xi l^{2}(l+1)^{2}-480 \mu^{2} l(l+1)+1920 \xi \mu^{2} l(l+1)\right)\right] \\
& +\ln \left(1+\frac{l(l+1)+2}{4 \mu^{2}}\right)\left[f \left(-360 \xi-960 \xi \mu^{2}-960 \xi \mu^{4}-360 \xi l(l+1)\right.\right. \\
& \left.\left.-60 \xi l^{2}(l+1)^{2}-480 \xi \mu^{2} l(l+1)\right)\right]+O\left(f^{2}\right),
\end{aligned}
$$

$$
\begin{aligned}
\left(192 \pi M^{2}\right) P= & 1-4 \mu^{2}-4 \xi-l(l+1)+16 \xi \mu^{2}+4 \xi l(l+1) \\
& +\ln \left(1+\frac{l(l+1)+1}{4 \mu^{2}}\right)\left[-6-24 \mu^{2}+24 \xi-6 l(l+1)+96 \xi \mu^{2}+24 \xi l(l+1)\right] \\
& +\ln \left(1+\frac{3 l(l+1)+2}{12 \mu^{2}}\right)\left[24 \mu^{2}-96 \xi \mu^{2}-24 \xi l(l+1)\right]+O(f) . \quad \quad \text { (4.11c) }
\end{aligned}
$$


The stress tensor and pressure for the conformal field are retrieved by taking $m, \xi$, and $l$ to zero [27].

The challenge remains to extend these explicit formulae for the stress tensor and pressure outside of the immediate neighborhood of the horizon, to match onto the high-frequency approximation $(3.16)$.

\section{CONCLUSIONS}

In this paper we have obtained an analytic approximation for the effective action of a quantum scalar field in a general static two-dimensional spacetime. The resulting expression shows clearly both the zero and the finite temperature contributions; these latter are competely missed in the construction based on functional integration of the conformal anomaly.

In this elegant and rather simple way an expression for the stress tensor of the scalar field can be obtained and is shown to coincide with the one obtained by standard canonical construction under the WKB approximation. The most remarkable aspect of this new method is that the same procedure can be repeated for the full $4 \mathrm{D}$ case [10, 11, 12], reproducing exactly the results of Anderson, Hiscock, and Samuel [16].

Unfortunately our effective action, like other analytical approaches, is unable to handle correctly the horizon region, where the high-frequency approximation breaks down. This causes the appearance of unphysical divergences in the stress tensor in the Hartle-Hawking state. We have shown how a careful analysis of the Green function near the horizon leads to the expected regular result.

Much effort is still needed to find an analytic expression for the stress tensor in black-hole spacetimes which correctly describes both the horizon and the asymptotic region. Only then can a serious analysis of nonperturbative backreaction effects in black-hole spacetimes start.

\section{Acknowledgments}

The authors are grateful to Valeri Frolov and Andrei Zelnikov for helpful discussions. PJS would like to thank the INFN and the Natural Sciences and Engineering Research Council of Canada for their financial support. This work has been funded by NSF grant PHY 00-99559 and its predecessor. The Center for Gravitational Wave Physics is supported by the NSF 
under co-operative agreement PHY 01-14375.

\section{APPENDIX A: WKB APPROXIMATION FOR THE $l=0$ MODE}

Here we outline the calculation of the stress tensor of the two-dimensional s-wave field $\widehat{\varphi}_{0}$ satisfying (2.4) with $l=0$ in the Schwarzschild spacetime (3.14) using the WKB approximation with point-splitting regularization. We show that the WKB approximation coincides exactly with the high-frequency approximation (3.16a, 3.16b) for $l=0$. These calculations closely follow analogous ones performed in [8]; details on the method can be found there and in the seminal paper by Anderson, Hiscock and Samuel [16].

To take advantage of the work of [8, 16] it is convenient to consider not the twodimensional Euclidean Green function $G$ of (2.9) but the rescaled quantity

$$
G_{E}\left(t, r ; t^{\prime}, r^{\prime}\right) \equiv r r^{\prime} G\left(t, r ; t^{\prime}, r^{\prime}\right)
$$

Substitution into (2.9) shows that $G_{E}$ satisfies

$$
\left[\nabla^{a}\left(e^{-2 \phi} \nabla_{a}\right)-m^{2} e^{-2 \phi}\right] G_{E}\left(x, x^{\prime}\right)=-\frac{\delta^{2}\left(x-x^{\prime}\right)}{\sqrt{h}},
$$

where $h_{a}^{c^{\prime}}$ is the bivector of parallel transport. The expressions (2.11a), 2.11d) for the unrenormalized point-split stress tensor become

$$
\begin{aligned}
\left\langle T_{a b}\left(x, x^{\prime}\right)\right\rangle= & e^{-\phi(x)-\phi\left(x^{\prime}\right)}\left\{\left(\frac{1}{2}-\xi\right)\left(h_{a}^{c^{\prime}} G_{E ; c^{\prime} b}+h_{b}^{c^{\prime}} G_{E ; a c^{\prime}}\right)+\left(2 \xi-\frac{1}{2}\right) h_{a b} h^{c d^{\prime}} G_{E ; c d^{\prime}}\right. \\
& \left.-\xi\left(G_{E ; a b}+h_{a}^{c^{\prime}} h_{b}^{d^{\prime}} G_{E ; c^{\prime} d^{\prime}}\right)+\left(2 \xi-\frac{1}{2}\right) h_{a b} m^{2} G_{E}\right\}
\end{aligned}
$$

We begin with the WKB approximation at zero temperature, and then calculate finitetemperature effects. We conclude by showing how to estimate the contribution of the zerofrequency mode at finite temperatures in flat space; this is useful for correcting the WKB and high-frequency approximations in asymptotically flat regions.

\section{Zero Temperature}

At zero temperature, this Green function has the integral expansion

$$
G_{E}\left(x, x^{\prime}\right)=\int_{0}^{\infty} \frac{d \omega}{4 \pi} \cos \left[\omega\left(t-t^{\prime}\right)\right] p_{\omega}\left(r_{<}\right) q_{\omega}\left(r_{>}\right),
$$


where $p_{\omega}$ and $q_{\omega}$ obey the differential equation

$$
f \frac{d^{2} S}{d r^{2}}+\left[\frac{2 f}{r}+f^{\prime}\right] \frac{d S}{d r}-\left(\frac{\omega^{2}}{f}+m^{2}\right) S=0 .
$$

One can write $p_{\omega}$ and $q_{\omega}$ in the WKB form

$$
\begin{aligned}
& p_{\omega}=\frac{1}{r \sqrt{2 W}} \exp \left\{\int^{r} d r \frac{W(r)}{f}\right\}, \\
& q_{\omega}=\frac{1}{r \sqrt{2 W}} \exp \left\{-\int^{r} d r \frac{W(r)}{f}\right\},
\end{aligned}
$$

where the function $W$ satisfies the equation

$$
W^{2}=\omega^{2}+m^{2} f+V+\frac{f}{2 W}\left[f \frac{d^{2} W}{d r^{2}}+\frac{d f}{d r} \frac{d W}{d r}-\frac{3 f}{2 W}\left(\frac{d W}{d r}\right)^{2}\right]
$$

with

$$
V=\frac{f}{r} \frac{d f}{d r} .
$$

This is solved iteratively starting with the zeroth-order solution

$$
W=\sqrt{\omega^{2}+m^{2} f} .
$$

In the following we omit terms coming from the conformally invariant part $(\xi=0, m=0)$, indicated with $\left\langle T_{a}^{b}\right\rangle^{0}$, which have been treated in [8]. We have

$$
\begin{aligned}
\left\langle T_{t}^{t}\right\rangle_{\text {unren }}= & \left\langle T_{t}^{t}\right\rangle_{\text {unren }}^{0}+\mathrm{e}^{-2 \phi} \int_{0}^{\infty} \frac{d \omega}{4 \pi} \cos \left(\omega \epsilon_{\tau}\right)\left\{-\frac{1}{2} m^{2} A_{1}+\xi\left[c_{0} \omega^{2} A_{1}+c_{1} A_{2}\right.\right. \\
& \left.\left.+c_{2} A_{3}+c_{3}\left(A_{4}+A_{5}\right)+c_{4} A_{5}+c_{5} A_{1}\right]\right\} \\
& +i \xi \mathrm{e}^{-2 \phi} \int_{0}^{\infty} \frac{d \omega}{4 \pi} \omega \sin \left(\omega \epsilon_{\tau}\right)\left[c_{6} A_{1}+c_{7}\left(A_{4}+A_{5}\right)\right], \\
\left\langle T_{r}^{r}\right\rangle_{\text {unren }}= & \left\langle T_{r}^{r}\right\rangle_{\text {unren }}^{0}+\mathrm{e}^{-2 \phi} \int_{0}^{\infty} \frac{d \omega}{4 \pi} \cos \left(\omega \epsilon_{\tau}\right)\left\{-\frac{1}{2} m^{2} A_{1}+\xi\left[c_{8} \omega^{2} A_{1}+c_{9} A_{3}\right.\right. \\
& \left.\left.+c_{10}\left(A_{4}+A_{5}\right)\right]\right\}+i \xi \mathrm{e}^{-2 \phi} \int_{0}^{\infty} \frac{d \omega}{4 \pi} \omega \sin \left(\omega \epsilon_{\tau}\right) c_{11} A_{1},
\end{aligned}
$$

where $\epsilon=t-t^{\prime}$ (for convenience the points are split in time only, so $r^{\prime}=r$ ), the $c_{i}$ at leading orders are

$$
\begin{aligned}
& c_{0}=\frac{2}{f}\left(1+\frac{M^{2} \epsilon^{2}}{r^{4}}\right), \quad c_{1}=2 h^{r r^{\prime}}, \quad c_{2}=O\left(\epsilon^{2}\right), \\
& c_{3}=-\frac{f^{\prime}}{2}, \quad c_{4}=O\left(\epsilon^{2}\right), \quad c_{5}=2 m^{2}, \\
& c_{6}=-h^{t t^{\prime}} h^{t r^{\prime}} f^{\prime}, \quad c_{7}=2 h^{r t^{\prime}}, \quad c_{8}=-\frac{M^{2} \epsilon^{2}}{f r^{4}}, \\
& c_{9}=O\left(\epsilon^{2}\right), \quad c_{10}=\left(\frac{2 f}{r}+\frac{f^{\prime}}{2}\right), \quad c_{11}=h^{r t^{\prime}} \frac{f^{\prime}}{f},
\end{aligned}
$$


and

$$
\begin{aligned}
& A_{1}=p_{\omega} q_{\omega}, \quad A_{2}=\frac{d p_{\omega}}{d r} \frac{d q_{\omega}}{d r}, \quad A_{3}=\frac{1}{4} p_{\omega} q_{\omega}, \\
& A_{4}=q_{\omega} \frac{d p_{\omega}}{d r}, \quad A_{5}=p_{\omega} \frac{d q_{\omega}}{d r} .
\end{aligned}
$$

The expansions for the bivector of parallel transport are

$$
\begin{aligned}
& h^{t t^{\prime}}=-\frac{1}{f}-\frac{f^{\prime 2}}{8 f} \epsilon^{2}+O\left(\epsilon^{4}\right), \\
& h^{t r^{\prime}}=-h^{r t^{\prime}}=-\frac{f^{\prime}}{2} \epsilon+O\left(\epsilon^{3}\right), \\
& h^{r r^{\prime}}=f+\frac{f^{\prime 2} f}{8} \epsilon^{2}+O\left(\epsilon^{4}\right) .
\end{aligned}
$$

Eventually one arrives at the following expression for $\left\langle T_{a}^{b}\right\rangle$ in the zero temperature state:

$$
\begin{aligned}
& \left\langle T_{t}^{t}\right\rangle_{\text {unren }}=\left\langle T_{t}^{t}\right\rangle_{\text {unren }}^{0}+\frac{m^{2}}{4 \pi} L+\frac{\xi}{2 \pi f}\left\{-\frac{4 M f}{r^{3}}-\frac{M^{2}}{r^{4}}+\left[\frac{2 M f}{r^{3}}-\frac{2 f^{2}}{r^{2}}\right] L\right\}, \\
& \left\langle T_{r}^{r}\right\rangle_{\text {unren }}=\left\langle T_{r}^{r}\right\rangle_{\text {unren }}^{0}+\frac{m^{2}}{4 \pi} L+\frac{\xi}{2 \pi f}\left\{\frac{M^{2}}{r^{4}}+\left[\frac{2 M f}{r^{3}}+\frac{4 f^{2}}{r^{2}}\right] L\right\},
\end{aligned}
$$

where $L=\left[\frac{1}{2} \ln \left(-\lambda^{2} \epsilon^{2}\right)+\gamma\right]$ and $\gamma$ is Euler's constant. To obtain the renormalized stress tensor, one subtracts from the above expression the renormalization counterterms obtained from the DeWitt-Schwinger Green function,

$$
G_{D S}=\frac{e^{\phi(x)+\phi\left(x^{\prime}\right)}}{2 \pi}\left\{-L^{\prime}\left[1+\alpha \sigma+O\left(\sigma^{2}\right)\right]+\frac{a_{1}}{2 m^{2}}+\beta \sigma+O\left(\sigma^{2}\right)\right\},
$$

where

$$
L^{\prime}=\frac{1}{2} \ln \frac{m^{2} \sigma}{2}+\gamma,
$$

$a_{1}$ is the DeWitt-Schwinger coefficient,

$$
a_{1}=\frac{1}{6}\left(R-6(\nabla \phi)^{2}+6 \square \phi\right),
$$

and

$$
\begin{aligned}
& \alpha=\frac{m^{2}}{2}+\frac{R}{12}-\frac{a_{1}}{2}, \\
& \beta=\frac{m^{2}}{2}-\frac{a_{1}}{4} .
\end{aligned}
$$

Recall that $R$ is the scalar curvature of the two-dimensional metric $h_{a b} ; \sigma$ is one-half the square of the geodesic distance between the points $x$ and $x^{\prime}$. Performing all the calculations, 
the counterterms read

$$
\begin{aligned}
& \left\langle T_{t}^{t}\right\rangle_{D S}=\left\langle T_{t}^{t}\right\rangle_{D S}^{0}+\frac{m^{2}}{4 \pi} L^{\prime}+\frac{\xi}{2 \pi f}\left\{\frac{2 M f}{r^{3}} L^{\prime}-\frac{2 f^{2}}{r^{2}} L^{\prime}\right\}+\frac{m^{2}}{8 \pi}, \\
& \left\langle T_{r}^{r}\right\rangle_{D S}=\left\langle T_{r}^{r}\right\rangle_{D S}^{0}+\frac{m^{2}}{4 \pi} L^{\prime}+\frac{\xi}{2 \pi f}\left\{-\frac{2 M f}{r^{3}}+\frac{2 M f}{r^{3}} L^{\prime}+\frac{4 f^{2}}{r^{2}} L^{\prime}\right\}-\frac{m^{2}}{8 \pi} .
\end{aligned}
$$

Subtracting these from the unrenormalized expressions (A.14a) one obtains the renormalized stress tensor in the Boulware vacuum:

$$
\begin{aligned}
\left\langle B\left|T_{t}^{t}\right| B\right\rangle_{\text {ren }}= & \left\langle B\left|T_{t}^{t}\right| B\right\rangle_{\text {ren }}^{0}-\frac{m^{2}}{8 \pi} \ln \left(\frac{m^{2} f}{4 \lambda^{2}}\right)+\frac{\xi}{2 \pi f}\left\{-\frac{4 M}{r^{3}}+\frac{7 M^{2}}{r^{4}}\right. \\
& \left.+\frac{f}{r^{2}}\left(1-\frac{3 M}{r}\right) \ln \left(\frac{m^{2} f}{4 \lambda^{2}}\right)\right\}-\frac{m^{2}}{8 \pi}, \\
\left\langle B\left|T_{r}^{r}\right| B\right\rangle_{\text {ren }}= & \left\langle B\left|T_{r}^{r}\right| B\right\rangle_{r e n}^{0}-\frac{m^{2}}{8 \pi} \ln \left(\frac{m^{2} f}{4 \lambda^{2}}\right)+\frac{\xi}{2 \pi f}\left\{\frac{2 M}{r^{3}}-\frac{3 M^{2}}{r^{4}}\right. \\
& \left.+\frac{f}{r^{2}}\left(\frac{3 M}{r}-2\right) \ln \left(\frac{m^{2} f}{4 \lambda^{2}}\right)\right\}+\frac{m^{2}}{8 \pi}
\end{aligned}
$$

where the conformal $(\xi=0, m=0)$ contribution is 8

$$
\begin{aligned}
& \left\langle B\left|T_{t}{ }^{t}\right| B\right\rangle=\frac{1}{2 \pi f}\left\{\frac{1}{12} \frac{M^{2}}{r^{4}}-\frac{1}{6} \frac{f M}{r^{3}}-\frac{f^{2}}{4 r^{2}} \ln \left(\frac{m^{2} f}{4 \lambda^{2}}\right)\right\}, \\
& \left\langle B\left|T_{r}{ }^{r}\right| B\right\rangle=\frac{1}{2 \pi f}\left\{-\frac{1}{12} \frac{M^{2}}{r^{4}}-\frac{1}{2} \frac{f M}{r^{3}}+\frac{f^{2}}{4 r^{2}} \ln \left(\frac{m^{2} f}{4 \lambda^{2}}\right)\right\} .
\end{aligned}
$$

\section{Finite-Temperature Corrections}

The thermal case is treated similarly. Evaluating the sum over discrete frequencies using the Plana sum formula [22] one finds that the stress tensor at finite temperature is obtained from the zero-temperature one by making the substitution

$$
\ln \left(\frac{m^{2} f}{4 \lambda^{2}}\right) \rightarrow\left\{2 \gamma+\ln \left(\frac{m^{2} \beta^{2} f}{16 \pi^{2}}\right)\right\}
$$

and adding the traceless pure radiation term

$$
\left\langle T_{t}^{t}\right\rangle_{\text {rad }}=-\left\langle T_{r}^{r}\right\rangle_{\text {rad }}=-\frac{\pi}{6 \beta^{2} f}
$$

where $\beta=T^{-1}$ is the inverse temperature. 


\section{Zero-Frequency Mode}

One can also use the WKB procedure to calculate the contribution of the $n=0$ mode at nonzero temperature in the asymptotically flat region, as mentioned in Section ПIIB. In this case the zeroth-order solution (A.9) for $W$ is sufficient,

$$
W=m \sqrt{f} .
$$

Substituting into (A.6) and using (A.11), (A.12) one can evaluate the contribution of this mode to the stress tensor, (A.10). Note that for a field at finite temperature, we should replace the integrals over continuous $\omega$ in (A.10) by sums. For the zero-frequency mode alone, it is sufficient to make the replacement

$$
\int \frac{d \omega}{4 \pi} F(\omega) \longrightarrow \frac{F(0)}{\beta}
$$

See, for example, the equations following (3.4) of [8]. Evaluating (A.10), we find that the only term in the stress tensor which is nonvanishing at spatial infinity is

$$
\left\langle T_{r}^{r}\right\rangle_{n=0}=-\frac{m}{2 \beta \sqrt{f}}
$$




\section{APPENDIX B: CORRECTED $G_{n}$ NEAR THE HORIZON}

The solutions to the differential equation (4.4) for the contribution $G_{n}$ to the twodimensional Green function for modes of small $n$ are

$$
\begin{aligned}
G_{0}\left(r, r^{\prime}\right)= & L_{<}^{\frac{1}{2}} I_{0}\left(\frac{\alpha_{0} L_{<}}{M}\right)\left(1-A_{0}^{2}\left(\frac{L_{<}}{4 M}\right)^{4}\right) \\
& \times L_{>}^{\frac{1}{2}}\left(K_{0}\left(\frac{\alpha_{0} L_{>}}{M}\right)-\frac{1}{2} A_{0}^{2}\left(\frac{L_{>}}{4 M}\right)^{4}\left[1-2 \gamma-2 \ln \left(\frac{\alpha_{0} L_{>}}{2 M}\right)\right]\right), \\
G_{1}\left(r, r^{\prime}\right)= & L_{<}^{\frac{1}{2}} I_{1}\left(\frac{\alpha_{1} L_{<}}{M}\right)\left(1-\frac{2}{3} A_{1}^{2}\left(\frac{L_{<}}{4 M}\right)^{4}\right) \\
& \times L_{>}^{\frac{1}{2}} K_{1}\left(\frac{\alpha_{1} L_{>}}{M}\right)\left(1-2 A_{1}^{2}\left(\frac{L_{>}}{4 M}\right)^{4}\right) \\
G_{2}\left(r, r^{\prime}\right)= & L_{<}^{\frac{1}{2}} I_{2}\left(\frac{\alpha_{2} L_{<}}{M}\right)\left(1-\frac{1}{2} A_{2}^{2}\left(\frac{L_{<}}{4 M}\right)^{4}\right) \\
& \times L_{>}^{\frac{1}{2}}\left(K_{2}\left(\frac{\alpha_{2} L_{>}}{M}\right)-\frac{1}{2 \alpha_{2}^{2}} A_{2}^{2}\left(\frac{L_{>}}{4 M}\right)^{2}\left[\gamma+\ln \left(\frac{\alpha_{2} L_{>}}{2 M}\right)\right]\right), \\
G_{3}\left(r, r^{\prime}\right)= & L_{<}^{\frac{1}{2}} I_{3}\left(\frac{\alpha_{3} L_{<}}{M}\right)\left(1-\frac{2}{5} A_{3}^{2}\left(\frac{L_{<}}{4 M}\right)^{4}\right) \\
& \times L_{>}^{\frac{1}{2}} K_{3}\left(\frac{\alpha_{3} L_{>}}{M}\right)\left(1+2 A_{3}^{2}\left(\frac{L_{>}}{4 M}\right)^{4}\right) .
\end{aligned}
$$

Here $L_{>}\left(L_{<}\right)$is the physical distance from the event horizon to the greater (lesser) of $r, r^{\prime}$ :

$$
L(r)=2 M\left[\sqrt{\frac{r}{2 M}\left(\frac{r}{2 M}-1\right)}+\ln \left(\sqrt{\frac{r}{2 M}}+\sqrt{\left.\frac{r}{2 M}-1\right)}\right] .\right.
$$

The expansions of each of these functions for small $f, f^{\prime}$ is significant up to and including the third term, and satisfies the differential equation (4.4) for the Green function to the order required for obtaining the stress tensor to $O\left(L^{\frac{1}{2}} \ln L\right)=O(f \ln f)$ inclusive. 
[1] S. W. Hawking, Commun. Math. Phys. 43, 199 (1975).

[2] V. Mukhanov, A. Wipf and A. Zelnikov, Phys. Lett. B 332, 283 (1994).

[3] S. Nojiri and S. D. Odintsov, Mod. Phys. Lett. A 12, 709 (1997).

[4] R. Bousso and S. Hawking, Phys. Rev. D 56, 7788 (1997).

[5] R. Balbinot and A. Fabbri, Phys. Rev. D 59, 044031 (1999); Phys. Lett. B 459, 112 (1999).

[6] W. Kummer and D. V. Vassilevich, Annalen Phys. 8, 801 (1999).

[7] M. Buric and V. Radovanovic, Phys. Rev. D 63, 044020 (2001).

[8] R. Balbinot, A. Fabbri, P. Nicolini, V. Frolov, P. Sutton, and A. Zelnikov, Phys. Rev. D 63, 084029 (2001).

[9] P. Sutton, Phys. Rev. D 62, 044033 (2000).

[10] P. Sutton, The Dimensional-Reduction Anomaly, Ph.D. thesis, University of Alberta (2000).

[11] V. Frolov, P. Sutton, and A. Zelnikov, Phys. Rev. D 61, 024021 (2000).

[12] V. Frolov, P. Sutton, and A. Zelnikov, in preparation (2002).

[13] S. M. Christensen, Phys. Rev. D 14, 2490 (1976); ibid. 17, 946 (1978).

[14] N. D. Birrell and P. C. W. Davies, Quantum fields in curved space (Cambridge, 1982).

[15] I. S. Gradshteyn and I. M. Ryzhik, Tables of Integrals, Series, and Products (Academic, New York, 1994).

[16] P. R. Anderson, W. A. Hiscock, and D. A. Samuel, Phys. Rev. D 51, 4337 (1995).

[17] V. P. Frolov, W. Israel, and S. N. Solodukhin, Phys. Rev. D 54, 2732 (1996).

[18] P. R. Anderson, Phys. Rev. D 41, 1152 (1990).

[19] D. N. Page, Phys. Rev. D 25, 1499 (1982); M. R. Brown and A. C. Ottewill, Phys. Rev. D 31, 2514 (1985); M. R. Brown, A. C. Ottewill, and D. N. Page, Phys. Rev. D 33, 2840 (1986).

[20] V. P. Frolov and A. I. Zel'nikov, Phys. Rev. D 35, 3031 (1987).

[21] P. Candelas and K. W. Howard, Phys. Rev. D 29, 1618 (1984), K. W. Howard and P. Candelas, Phys. Rev. Lett. 53, 403 (1984); K. W. Howard, Phys. Rev. D 30, 2532 (1984).

[22] G. Chika and M. Visinescu, Nuovo Cimento A46, 25 (1978); E. T. Whittaker and G. N. Watson, A Course of Modern Analysis (Cambridge University Press, Cambridge, England, 1927), p. 145 , exercise 7 .

[23] A static geometry is a requirement of our approximation scheme. 
[24] There is an ambiguity in how one specifies the divergent part of the logarithm term in (3.2). Our choice corresponds to using $\ln \frac{m^{2}\left(\tau^{2}+\epsilon^{2}\right)}{4 \mathrm{e}^{-2 \gamma}} \rightarrow \ln \frac{m^{2}\left(\tau^{2}+\epsilon^{2}\right)}{1+m^{2} \tau^{2}}$.

[25] $L\left(x, x^{\prime} \mid \omega\right)$ also contains a $1 / \epsilon$ term which is the usual leading divergence for a one-dimensional action; it is the 'superficial' divergence encountered by Candelas and Howard [21] and others [16, 18], and may be safely ignored.

[26] The stress tensor will be finite in a freely-falling frame on the past and future event horizons provided that as $r \rightarrow 2 M$

$$
\left|T_{t}^{t}+T_{r}^{r}\right|<\infty, \quad f^{-1}\left|T_{t}^{t}-T_{r}^{r}\right|<\infty, \quad|P|<\infty
$$

[27] An error was made in equation (4.16) of [8] which affected the finite terms in (4.20), (4.21), and (4.23) of that paper. Equation (4.16) should read

$$
\left\langle T_{a}^{b}\right\rangle_{0}=O\left(f^{2}\right)
$$

As a consequence, in (4.20) the factor $17 / 240$ should be replaced by $1 / 24$, in (4.21) the factor $-83 / 960$ should be replaced by $-11 / 192$, and in (4.23) the factor $-23 / 240$ should be replaced by $1 / 48$. The conclusions regarding the regularity of the field at the horizon are unchanged. Note also that in [8] $P$ was defined with an additional factor of $\left(4 \pi r^{2}\right)^{-1}$. 